\title{
ELISA Saliva for Trypanosoma cruzi Antibody Detection: An Alternative for Serological Surveys in Endemic Regions
}

\author{
Léa Campos de Oliveira, ${ }^{1}$ Natalia Bueno Pereira, ${ }^{2}$ Carlos Henrique Valente Moreira, ${ }^{2,3}$ Ana Luiza Bierrenbach, ${ }^{4}$ \\ Flavia Cristina Salles, ${ }^{2}$ Marcela de Souza-Basqueira, ${ }^{2}$ Erika Regina Manuli, ${ }^{2}$ Ariela Mota Ferreira, ${ }^{5}$ Cláudia Di Lorenzo Oliveira, ${ }^{6}$ \\ Clareci Silva Cardoso, ${ }^{6}$ Antonio Luiz P. Ribeiro, ${ }^{7}$ and Ester Cerdeira Sabino ${ }^{2 *}$ \\ ${ }^{1}$ LIM03, Hospital das Clínicas, Medical School, University of São Paulo, São Paulo, Brazil; ${ }^{2}$ School of Medicine, University of São Paulo, São Paulo, \\ Brazil; ${ }^{3}$ Institute of Infectology Emílio Ribas, São Paulo, Brazil; ${ }^{4}$ Teaching and Research Institute, Hospital Sírio-Libanês, São Paulo, Brazil; ${ }^{5}$ State \\ University of Montes Claros, Montes Claros, Brazil; ${ }^{6}$ Federal University of São João del-Rei, Divinópolis, Minas Gerais, Brazil; ${ }^{7}$ Hospital das Clínicas \\ and School of Medicine, Universidade Federal de Minas Gerais, Belo Horizonte, Brazil
}

\begin{abstract}
Chagas is a neglected disease endemic in Latin America. Vector transmission control had been aggressively performed. Recent entomological surveillance in Brazil has revealed natural infection rates ranging from $0.40 \%$ to $0.52 \%$. Although serological surveys are complex to develop, they are important for disease control. In this study, we validated the use of saliva in ELISA commercial kits with a cohort of 100 patients with Chagas disease followed at Hospital das Clinicas in São Paulo, Brazil, and 50 healthy controls. Five ELISA kits for detecting antibodies against Trypanosoma cruzi were tested. The best discrimination between Chagas patients and controls was observed with the Wiener kit, which yielded a sensitivity of $97 \%$ and a specificity of $100 \%$. Our findings reveal that the use of saliva may be an alternative to large-scale screening surveys in detecting $T$. cruzi antibodies; it is a noninvasive sample collection method potentially key to large-scale screening in children.
\end{abstract}

\section{INTRODUCTION}

Chagas disease $(\mathrm{ChD})$ is caused by the protozoan Trypanosoma cruzi and is naturally transmitted by insects from Reduviidae family. The disease may also be acquired congenitally, by organ transplantations or blood transfusions. ${ }^{1,2}$ Chagas disease is endemic in Latin America, and 70 million people are at risk of becoming infected. Because of effective domestic vector control and screening of blood donors for $T$. cruzi being carried out in most Latin American countries, most of the current cases are in indeterminate and chronic forms of the disease. ${ }^{3}$ There are 5.7 million infected people in Latin America, ${ }^{4}$ with $70,000 \mathrm{ChD}$-related deaths annually in the region and 528,000 disability-adjusted life years. ${ }^{5}$ In Brazil, it is estimated that there are 2-3 million infected individuals. ${ }^{6}$

Because of globalization and increasing international travel, ChD cases have also been reported in Europe and Asia. ${ }^{7}$ Around $99 \%$ of the millions of infected people have poor or no access to medical care and remain undiagnosed and untreated. ${ }^{8}$

Despite the main vector, Triatoma infestans have been totally controlled, other peridomestic vectors may transmit the parasite in Brazil. Residual infestation and recolonization of households occur in endemic areas, threatening the long-term success of vector control. ${ }^{9}$ Nevertheless, in our recent study, $46 \%$ of participants related the presence of "kissing bug" in their homes (unpublished data, E. C. Sabino). Chronic limitations in personnel and resources of the health sector in Brazilian rural areas, associated with new public health threats such as dengue, Zika, and chikungunya, pose serious obstacles to maintaining the Chagas vector-control programs.

Surveillance of large territorial areas is not simple. Therefore, in this study, we evaluated a simple, noninvasive,

\footnotetext{
*Address correspondence to Ester Cerdeira Sabino, School of Medicine, University of São Paulo, Av. Dr. Enéas Carvalho de Aguiar, 470, Cerqueira Cesar, São Paulo 0540300, Brazil. E-mail: sabinoec@ gmail.com
}

saliva-screening tool as an alternative for serological surveys in endemic regions.

\section{MATERIALS AND METHODS}

Samples. One hundred T. cruzi-infected patients, followed at Hospital das Clinicas de São Paulo, Brazil, and 50 healthy controls were enrolled in the study. We collected whole saliva samples in plastic tubes (Salivette, Sarstedt, Nümbrecht, Germany) according to the manufacturer's instructions and stored at $-20^{\circ} \mathrm{C}$ until use.

ELISA kits. Five commercial ELISA kits for detecting antibodies against $T$. cruzi were tested: ARCHITECT Chagas (Abbott, Chicago, IL), ELISA Chagas REC (Bio-Manguinhos, Rio de Janeiro, Brazil), Kit test Gold ELISA Chagas (REM, Sao Paulo, Brazil), Kit Chagatest-ELISA recombinant v.4.0 (Wiener, Rosario, Argentine), and Kit Test ELISA Chagas III-(Grupo Bios S.A-Diasorin, Santiago, Chile). The procedures were adapted for saliva use when possible.

ARCHITECT Chagas. All the tests were performed according to the manufacturer's instructions. The equipment did not allow for any modifications.

ELISA Chagas REC. These tests were performed according to the manufacturer's instructions with the following modifications: $100 \mu \mathrm{L}$ and $200 \mu \mathrm{L}$ whole saliva and $100 \mu \mathrm{L}$ and $200 \mu \mathrm{L}$ of peroxidase-conjugated anti-human lgG (kit).

Gold ELISA Chagas. These tests were performed according to the manufacturer's instructions with the following modifications: $220 \mu \mathrm{L}$ and $300 \mu \mathrm{L}$ whole saliva and $200 \mu \mathrm{L}$ of peroxidaseconjugated anti-human IgG (kit).

Test ELISA Chagas III. These tests were performed according to the manufacturer's instructions with the following modifications: $220 \mu \mathrm{L}$ of whole saliva, $100 \mu \mathrm{L}$ or $200 \mu \mathrm{L}$ of peroxidase-conjugated anti-human IgG (kit), $100 \mu \mathrm{L}$ of peroxidase-conjugated anti-human IgG (kit), and $100 \mu \mathrm{L}$ of horseradish peroxidase (HRP)-conjugated rabbit anti-human IgGP0214 (1:500 and 1:1000, DAKO Cytomation, Glostrup, Denmark) or $100 \mu \mathrm{L}$ of HRP-conjugated polyclonal goat antirabbit antibody (1:500, DAKO Cytomation). 
Chagatest-ELISA recombinant v.4.0. These tests were performed according to the manufacturer's instructions with the following modifications: $200 \mu \mathrm{L}$ of whole saliva and $100 \mu \mathrm{L}$ of pure peroxidase-conjugated anti-human IgG without dilution and incubated for 60 minutes.

\section{RESULTS}

Initial tests were performed with samples from 10 seropositive $T$. cruzi patients and five healthy controls using the five commercial ELISA kits. These results are presented in Figure 1. The best discrimination between positive and negative saliva antibodies against $T$. cruzi was observed with the ELISA-Kit Chagatest-ELISA recombinant v.4.0-Wiener. Therefore, we selected this kit for testing all of the samples.

The cutoff of 0.391 for saliva with Chagatest was established as two SDs above the mean absorbance of saliva from 50 healthy controls. The sensitivity of this test was $97 \%$ and the specificity $100 \%$. We also analyzed using the receiver operating characteristic curve (Figure 2); the obtained cutoff (0.389) was similar to the previous one, and the sensitivity and specificity were $98 \%$ and $100 \%$, respectively. There was a good discrimination between negative and positive samples (Figure 3).

In a subsample of five Chagas cases, we also analyzed the preservation of antibodies in the saliva samples stored at room temperature for 5 days compared with those processed immediately after collection. No differences were observed between sample-processing methods.

\section{DISCUSSION}

In this study, we evaluated an alternative method for largescale $T$. cruzi antibody screening, especially among children, using saliva samples instead of blood samples to detect antibodies to $T$. cruzi in commercial ELISA kits.

Saliva specimens provide interesting advances for diagnosis because their collection is noninvasive and, therefore, well accepted by patients. In addition, collecting these samples does not necessitate medically trained staff, and the samples are easy to process. Studies have demonstrated that saliva samples are interesting alternatives to venous blood specimens for different diseases, including infectious diseases, such as dengue and Hepatitis E virus (HEV), particularly in situations when blood collection is difficult. ${ }^{10,11}$

Saliva samples also appear to be highly reliable for the diagnosis of visceral leishmaniasis; they yield fewer falsepositive results than serum samples. Other studies have highlighted the usefulness of noninvasive samples such as urine and saliva as diagnostic tools for patients with kala azar, particularly in endemic regions. ${ }^{12}$

In addition, studies have suggested that salivary antibody responses can be used to detect incident infections with
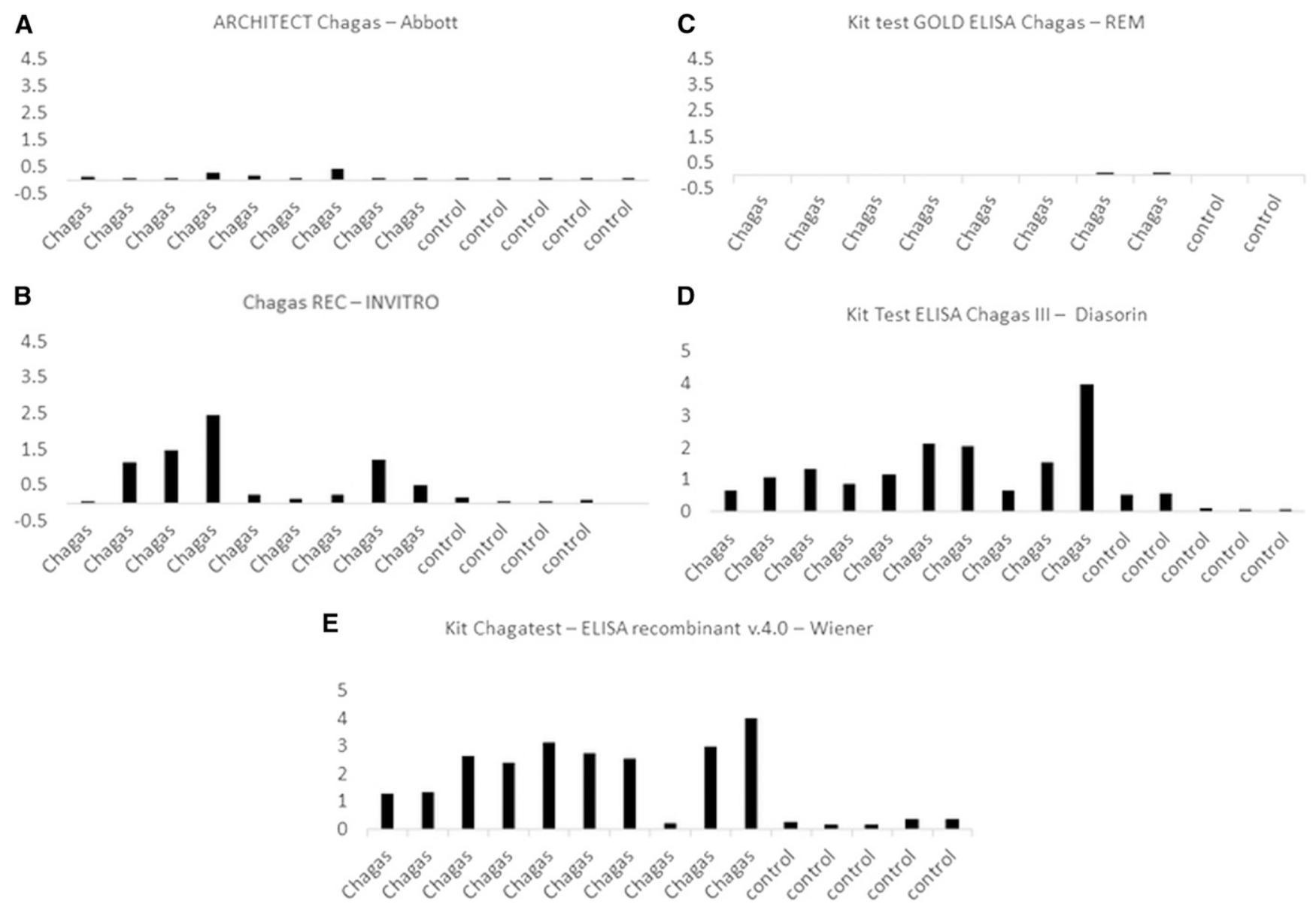

FIGURE 1. Antibody detection in saliva samples of patients with Chagas disease and health controls in different commercial ELISA kits. (A) Abbott; (B) in vitro; (C) Gold; (D) Diasorin; (E) Wiener. 


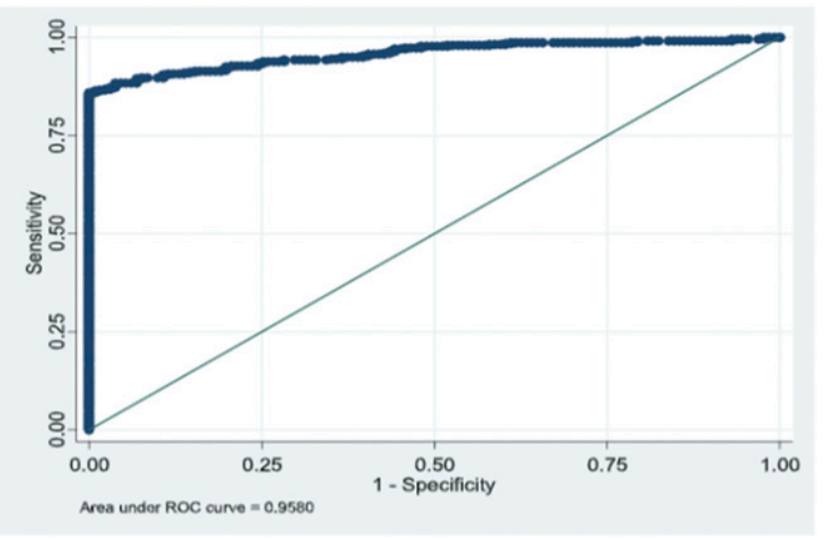

FIGURE 2. Area under receiver operating characteristic curve of Kit Chagastest-ELISA recombinant v.4.0 using saliva samples instead of serum for the detection of antibodies against Trypanosoma cruzi. This figure appears in color at www.ajtmh.org.

norwalk virus in prospective surveys. ${ }^{13}$ Also for $\mathrm{ChD}$, an inhouse method was developed, but the sensitivity was small for epidemiological surveys. ${ }^{14}$ Recently, a study similar to ours analyzing two commercial kits shows that it is a good alternative for Chagas screening, but the number of samples included in that study was a quite low. ${ }^{15}$

The assay developed in our laboratory using saliva has the potential to be beneficial outside a hospital environment in remote, resource-limited locations.

A possible limitation to this kind of study could be how well saliva samples are preserved after collection. In this pilot study with a small number of samples, we observed that the saliva is preserved for up to 5 days at room temperature. There is also a concern about the validation of the sensitivity for this test that must be replicated with a larger number of positive samples. Moreover, the fact that the sensitivity of saliva specimen tests is high (98\%) but not $100 \%$ compared with blood specimen tests indicates the need to correct this problem in epidemiological survey analyses.

Oral fluid and urine rapid diagnostic tests offer an attractive apparent alternative option to blood for diagnosis. A series of

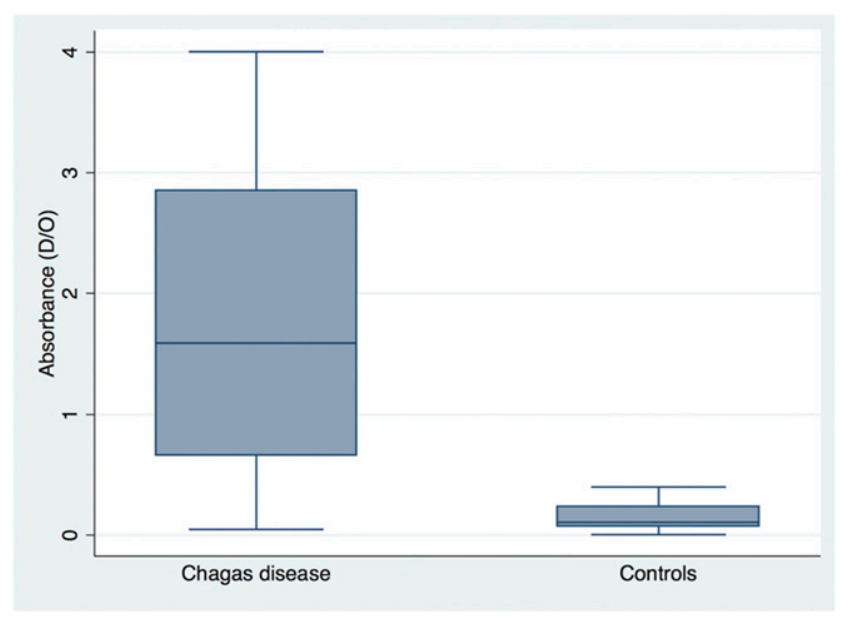

Figure 3. Box plot of antibodies against Trypanosoma cruzi comparing health controls with Chagasic patients. This figure appears in color at www.ajtmh.org. diagnostic tools has been developed by a commercial company, but such tools require further improvement, particularly in a context in which bodily fluids are already known to perform more poorly than blood specimens. ${ }^{16}$

The ability to use noninvasive techniques to assess patients rapidly at the point of care is a major goal of modern medicine. Therefore, saliva is an attractive bio-fluid to assess health, disease, and development. Saliva is preferred over other types of biological samples because of its convenience and ease of collection, and it may be collected repeatedly, even in the most vulnerable patients, without risk of harm. Recent technological advancements have improved our ability to detect thousands of proteins and/or microbes from a single salivary sample, making noninvasive assessment in neonates possible. ${ }^{17}$

Saliva presents a large number of analytes that may be affected by diverse physiological and pathological conditions. Furthermore, the noninvasive, simple, and cost-effective collection methods prompt interest in evaluating the diagnostic and prognostic utility of saliva. However, saliva should be carefully evaluated in relation to pre-analytical and analytical variables such as collection and storage methods, analyte circadian variation, sample recovery, prevention of sample contamination, and analytical procedures.

Because of its antioxidant status, saliva has also been used to measure oxidative stress in individuals with type 2 diabetes. ${ }^{18}$ Furthermore, several studies have demonstrated correlations of salivary markers of stress and insulin resistance including cortisol, insulin, adiponectin, and resistin with serum concentrations. These findings suggest the potential diagnostic value of saliva in health screening and risk stratification studies, particularly in pediatric populations, with implications for inflammatory, metabolic, and cardiovascular conditions. ${ }^{18}$

Therefore, using saliva as a fluid for diagnostic purposes in $\mathrm{ChD}$ would be a huge breakthrough for both patients and healthcare providers because saliva collection is easy, noninvasive, and inexpensive. Different diagnostic applications of saliva shed light on emerging technologies and tools for disease screening, detection, and monitoring. Moreover, the use of a noninvasive sample collection method will be key for applying large-scale screening in children.

Received April 17, 2018. Accepted for publication January 17, 2020.

Published online February 24, 2020.

Authors' addresses: Léa Campos de Oliveira, LIM03, Hospital das Clínicas, Medical School, University of São Paulo, São Paulo, Brazil, E-mail: lea.c.oliveira@gmail.com. Natalia Bueno Pereira, Flavia Cristina Salles, Marcela de Souza-Basqueira, Erika Regina Manuli, and Ester Cerdeira Sabino, School of Medicine, University of São Paulo, São Paulo, Brazil, E-mails: nataliabp2@hotmail.com, flaviacrissalles@ bol.com.br, marceladesouza88@gmail.com, erikamanuli@gmail.com, and sabinoec@gmail.com. Carlos Henrique Valente Moreira, School of Medicine, University of São Paulo, São Paulo, Brazil, and Institute of Infectology Emílio Ribas, São Paulo, Brazil, E-mail: carloshvmoreira@ gmail.com. Ana Luiza Bierrenbach, Teaching and Research Institute, Hospital Sírio-Libanês, São Paulo, Brazil, E-mail: carloshvmoreira@ gmail.com. Ariela Mota Ferreira, State University of Montes Claros, Montes Claros, Brazil, E-mail: arielamota@hotmail.com. Cláudia Di Lorenzo Oliveira and Clareci Silva Cardoso, Federal University of São João del-Rei, Brazil, E-mails: claudia.dlorenzo@gmail.com and clarecicardoso@yahoo.com.br. Antonio Luiz P. Ribeiro, Hospital das Clínicas and School of Medicine, Universidade Federal de Minas Gerais, Belo Horizonte, Brazil, E-mail: tom1963br@yahoo.com.br. 


\section{REFERENCES}

1. Howard EJ, Xiong X, Carlier Y, Sosa-Estani S, Buekens P, 2014. Frequency of the congenital transmission of Trypanosoma cruzi: a systematic review and meta-analysis. BJOG 121: 22-33.

2. Kransdorf EP, Zakowski PC, Kobashigawa JA, 2014. Chagas disease in solid organ and heart transplantation. Curr Opin Infect Dis 27: 418-424.

3. Rassi A, Marin-Neto JA, 2010. Chagas disease. Lancet 375: 1388-1402.

4. Dias JC, Silveira AC, Schofield CJ, 2002. The impact of Chagas disease control.in Latin America: a review. Mem Inst Oswaldo Cruz 97: 603-612.

5. WHO, 2015. Investing to Overcome the Global Impact of Neglected Tropical diseases. Third WHO Report on Neglected Tropical Diseases. Geneva, Switzerland: World Health Organization.

6. Martins-Melo FR, Ramos AN, Alencar CH, Heukelbach J, 2014. Prevalence of Chagas disease in Brazil: a systematic review and meta-analysis. Acta Trop 130: 167-174.

7. Gascon J, Bern C, Pinazo MJ, 2010. Chagas disease in Spain, the United States and other non-endemic countries. Acta Trop 115: 22-27.

8. Drugs for Neglected Diseases initiative, 2014. The Global Chagas Disease Coalition stages high-level event to encourage increased access to diagnosis and treatment of patients fighting Chagas disease [press release]. Mexico City, Mexico. 11 August 2014.

9. Schofield CJ, Jannin J, Salvatella R, 2006. The future of Chagas disease control. Trends Parasitol 22: 583-588.
10. Andries AC, Duong V, Ong S, Ros S, Sakuntabhai A, Horwood P, Dussart P, Buchy P, 2016. Evaluation of the performances of six commercial kits designed for dengue NS1 and anti-dengue $\lg \mathrm{M}, \lg \mathrm{G}$ and $\lg \mathrm{A}$ detection in urine and saliva clinical specimens. BMC Infect Dis 16: 201.

11. Singh MP, Majumdar M, Goyal K, Lakshmi PV, Bhatia D, Ratho RK, 2016. Investigation of suspected viral hepatitis outbreaks in North West India. Diagn Microbiol Infect Dis 84: 309-314.

12. Mohapatra S, Samantaray JC, Ghosh A, 2016. A comparative study of serum, urine and saliva using rk39 strip for the diagnosis of visceral leishmaniasis. J Arthropod Borne Dis 10: 87-91.

13. Griffin SM, Converse RR, Leon JS, Wade TJ, Jiang X, Moe CL, Egorov Al, 2015. Application of salivary antibody immunoassays for the detection of incident infections with Norwalk virus in a group of volunteers. J Immunol Methods 424: 53-63.

14. Pinho RT, Pedrosa RC, Costa-Martins P, Castello-Branco LR, 1999. Saliva ELISA: a method for the diagnosis of chronic Chagas disease in endemic areas. Acta Trop 72: 31-38.

15. Cortes-Serra N, Pinazo MJ, de la Torre L, Galizzi M, Gascon J, Bustamante JM, 2018. Diagnosis of Trypanosoma cruzi infection status using saliva of infected subjects. Am J Trop Med Hyg 98: 464-467.

16. Nunes LA, Mussavira S, Bindhu OS, 2015. Clinical and diagnostic utility of saliva as a non-invasive diagnostic fluid: a systematic review. Biochem Med (Zagreb) 25: 177-192.

17. Khanna $P$, Walt DR, 2015. Salivary diagnostics using a portable point-of-service platform: a review. Clin Ther 37: 498-504.

18. Desai GS, Mathews ST, 2014. Saliva as a non-invasive diagnostic tool for inflammation and insulin-resistance. World J Diabetes 5: 730-738. 